\title{
Nasal Bone Length Normogram of Mid-second Trimester Euploid Fetuses of an Indian Population
}

\author{
${ }^{1}$ Seneesh Kumar Vikraman, ${ }^{2}$ Vipin Chandra, ${ }^{3}$ Bijoy Balakrishanan, ${ }^{4}$ Amruta Gudi, ${ }^{5}$ Meenu Batra, ${ }^{6}$ Gopinathan Kannoli
}

\begin{abstract}
Introduction: Down syndrome (DS) is the most common chromosomal aneuploidy to result in a live birth. Absent nasal bone has been consistently observed in fetuses with DS. Multiple studies have been conducted to measure and compare the nasal bone length (NBL) in different races and ethnicity and is found to vary in size. In countries, such as India where often only the second trimester scan is performed for screening of aneuploidies, NBL could be regarded as a useful marker.
\end{abstract}

Aims and objectives: To estimate the NBL of fetuses at 18 to $22+6$ weeks gestational age in an Indian population, establish a normogram and compare it with that of the Caucasian population.

Materials and methods: Prospective observational study in a tertiary care hospital with 500 antenatal subjects after considering the inclusion and exclusion criteria.

Results: The normogram was established and found to be comparable to previously established ones for Indian subjects. The 5th centiles for 18-18.6, 19-19.6, 20-20.6, 21-21.6 and 2222.6 weeks of gestations were $3.4,3.5,4.2,4.3$ and $5.4 \mathrm{~mm}$ respectively. The NBL increased with gestational age (GA), and the mean NBL was $5.48 \mathrm{~mm}$. No correlation was noted with maternal age, sex of fetus or religion. The NBLs were significantly shorter than those of the Caucasian population.

Conclusion: Mean NBL values of Indian population are less than the mean NBL of Caucasian population. This along with the GA needs to be considered in counseling patients and decisions regarding further invasive and noninvasive tests.

Keywords: Normogram, Nasal bone length, Caucasian, Down syndrome, Correlation, Regression.

How to cite this article: Vikraman SK, Chandra V, Balakrishanan B, Gudi A, Batra M, Kannoli G. Nasal Bone Length Normogram of Mid-second Trimester Euploid Fetuses of an Indian Population. Int J Infertil Fetal Med 2015;6(1):25-29.

Source of support: Nil

Conflict of interest: None

Date of Received: $10-02-15$

Date of Acceptance: 14-03-15

Date of Publication: April 2015

\footnotetext{
1-3,5,6 Consultant, ${ }^{4}$ DNB Resident

1-3,5,6 Department of Fetomaternal Medicine, Edappal Hospitals Private Ltd, Malappuram, Kerala, India

${ }^{4}$ Department of Obstetrics and Gynecology, Edappal Hospitals Private Ltd, Malappuram, Kerala, India
}

Corresponding Author: Seneesh Kumar Vikraman, Consultant Department of Fetomaternal Medicine, Edappal Hospitals Private Ltd, Malappuram, Kerala, India, Phone: 04942660304, e-mail: drseneeshkv@gmail.com

\section{INTRODUCTION}

Down syndrome (DS) is the most common chromosomal aneuploidy to result in a live birth. ${ }^{1}$ Prenatal diagnosis can be performed using variety of sonographic markers. Absent nasal bone has been consistently observed in fetuses with DS.

Nasal bone has been shown to be absent in $73 \%$ of fetuses with DS in the first trimester, compared with $8.3 \%$ of control fetuses in Caucasian population. ${ }^{2}$ Nuchal translucency and absent nasal bone are now established aneuploidy markers for DS in the first trimester. ${ }^{3,4}$ Other first trimester aneuploidy markers include abnormal flow velocity patterns in the Ductus venosus, maxillary hypoplasia, frontomaxillary facial (FMF) angle and tricuspid regurgitation. ${ }^{5}$

In the second trimester identification of aneuploid fetuses is dependent mainly on sonographic markers such as a thickened Nuchal skin fold, long-bone length, pyelectasis, echogenic intracardiac foci and hyperechoic bowel. ${ }^{6}$ Absent or hypoplastic nasal bone length (NBL) has been described as a marker for trisomy 21 at 15 to 22 weeks gestational age (GA) with a likelihood ratio of 50 to $83^{2,7}$ and a sensitivity of $60 \%$ at a FPR of 1 to $5 \%$, making it as sensitive as the maternal serum biochemistry in detecting DS in the second trimester. ${ }^{7,8}$

Multiple studies have been conducted to measure and compare the NBL in different races and ethnicity and is found to vary in size. ${ }^{9}$ Very little systematic studies has been done on NBL in the second trimester as a marker for DS in the Indian Population. The Indian NBLs falls below the average on the Caucasian reference curves often enough to warrant development of indigenous normal curves of NBLs. ${ }^{10}$

In this study, we seek to evaluate the normal values of NBL in fetuses of 18 to $22+6$ weeks GA and to establish its normal range in a south Indian population by using two-dimensional (2D) ultrasound and compare it with that of a Caucasian population.

\section{MATERIALS AND METHODS}

This is a prospective observational study conducted in Edappal hospitals Pvt Ltd, Edappal, India, in the Division of Fetomaternal Medicine under Obstetrics and Gynecology Department, for 1 year from November 1st 2012 to October 30th 2013. 
Initially, approval from institutional ethical committee was obtained. The inclusion criteria were pregnant Indian women with singleton pregnancies in second trimester $(18-22+6$ weeks $)$ in the age group 17 to 35 years with excellent dates confirmed with a dating scan and willing to get enrolled. Gestational age was calculated by the last menstrual period, if the discrepancy between it and GA calculated from crown rump length (CRL) was less than 6 days. If discrepancy $\geq 7$ days, GA was corrected using CRL.

The exclusion criteria were pregnant women of age $<17$ or $>35$ years, multiple gestations, previous history of chromosomal abnormality, referral because of suspected fetal abnormality by a previous ultrasound examination and presence of structural abnormality in fetus.

After considering the inclusion and exclusion criteria, 500 consecutive antenatal subjects married to Indian partners with singleton pregnancies attending the Fetomaternal department for a Target scan (18-22 weeks) were included and followed up in the study. Most of the women were from the IVF clinic of this center. Informed consent was obtained from all individual participants included in the study. The sample size of 500 was chosen considering $95 \%$ confidence level and 4.5 confidence interval. The gestational window of 18 to $22+6$ was chosen as this constitutes the bulk of population attending the target scan and in a substantial subset their first scan in the Indian scenario. The Medical termination of pregnancy act in India its current form with the proposed amendment of raising upper limit to 24 weeks limits the usefulness of screening strategies beyond 23 to 24 weeks.

Pregnancies were followed up for their outcomes and those women with a confirmed normal outcome without structural or chromosomal abnormalities (Euploid and non-malformed) were included for analysis.

The image of the fetal facial profile were taken in the midsagittal plane after write zoom so that head and upper thorax occupied 2/3rd of the screen and tips of the nose, lips and chin were visualized. The angle of insonation was around $45^{\circ}$ to a line passing along to anterior aspect of frontal bone. At this angle ultrasound beams are perpendicular to nasal bone. The nasal bone was identified as a discrete thin echogenic line (Fig. 1). The NBL was measured from the base of the nose closest to the frontal bones to the farthest extent of ossification on the nose.

The fetal target scan was performed by an experienced fetomaternal medicine consultant on GE Voluson 730 Expert (GE Healthcare, Vienna, Austria) ultrasound machine using 3 to $5 \mathrm{MHz} 2 \mathrm{D}$ curvilinear transducer.

Nasal bone length was obtained thrice and its mean was taken. A permanent record of the image was retained in digital format for future review. The data were entered into a case record form and was analyzed in detail to assess the outcome measures. The results were assessed by plotting the NBL distribution in centiles at each gestational age. Correlation and regression analysis using NBL as the dependent variable and GA as independent variables was performed.

Salient features of the regression analysis used for this study are as follows:

1. The regression analysis was performed using SPSS statistical software 20.

2. The R-square value obtained was 0.392. It suggests that $39.2 \%$ of the variation of the NBL values can be explained by GA Variation.

3. The $F$ value is 321.7 and sig $F$ value is $<0.001$. It suggests

a. The Variables, i.e. GA and NBL are linearly correlated.

b. The Sig F Value is less than $0.001 \%$, it suggests that there is $99.9 \%$ possibility that the relationship among the NBL and GA is not due to chance.

4. The regression equation derived, can help determine the value of NBL (Dependant variable), given the value of GA (Independent variable).

\section{RESULTS}

All 500 antenatal subjects belonged to south Indian population. The study was conducted from November 1, 2012 to October 30, 2013. Where appropriate the results were compared with a similar Indian study by Sharma et $\mathrm{al}^{10}$ and studies on Caucasian population by Sonek et $\mathrm{al}^{11}$ and Gamez et al. ${ }^{12}$

Of the 500 subjects, the major proportion of the subjects were in the GA 20 to 20.6 weeks and 21 to 21.6 weeks (32.2 and $33.8 \%$ respectively). The mean GA was 20 weeks 6 days. $40.8 \%$ of the subjects were in the age group 21 to 25 and $32.2 \%$ in the age group 26 to 30 years. The age

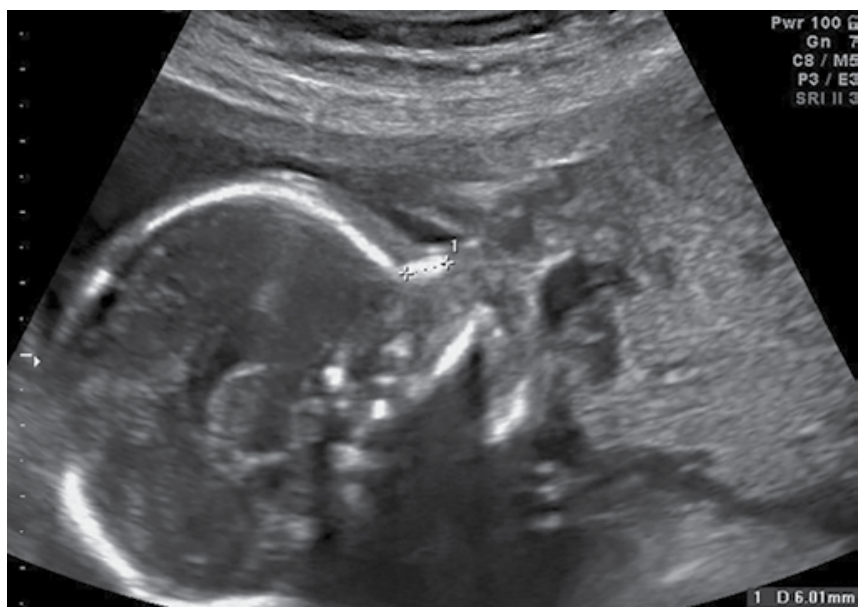

Fig. 1: Representative sonogram for nasal bone length (NBL) measurement: a mid-sagittal view of the face with the nasal bones appearing as a linear echogenic structure. The NBL was measured from the base of the nose closest to the frontal bones to the farthest extent of ossification on the nose. The appropriate caliper placement for NBL measurement is shown 
group 17 to 20 years had the least number of subjects $(11.6 \%)$. The mean maternal age was 25.7 years. There was no correlation between maternal age and NBL.

The NBL increased with GA, and the mean NBL was $5.48 \mathrm{~mm}$. A scatter plot was plotted with GA and means NBL measurements (Graph 1). It showed a line with R2 value of 0.392 suggestive of a positive correlation between GA and NBL.

A comparison of the NBL in the respective gestational ages from 18 to 22 weeks with the sex of the fetuses was performed and the $\mathrm{p}$-value were obtained by analysis of variance (ANOVA). p-values were 0.694, 0.896, 0.324, 0.714 and 0.039 respectively for 18, 19, 20, 21 and 22 weeksshowing that there is no statistical significant difference in the NBL in male and female fetuses at 18 to 21 weeks, except at 22 to 22.6 weeks with male NBL being slightly more compared to the female NBL.

The observed values of NBL in the study subjects were systematically analyzed. The results were tabulated (Tables 1 and 2) and line diagrams were drawn (Graphs 2 and 3).

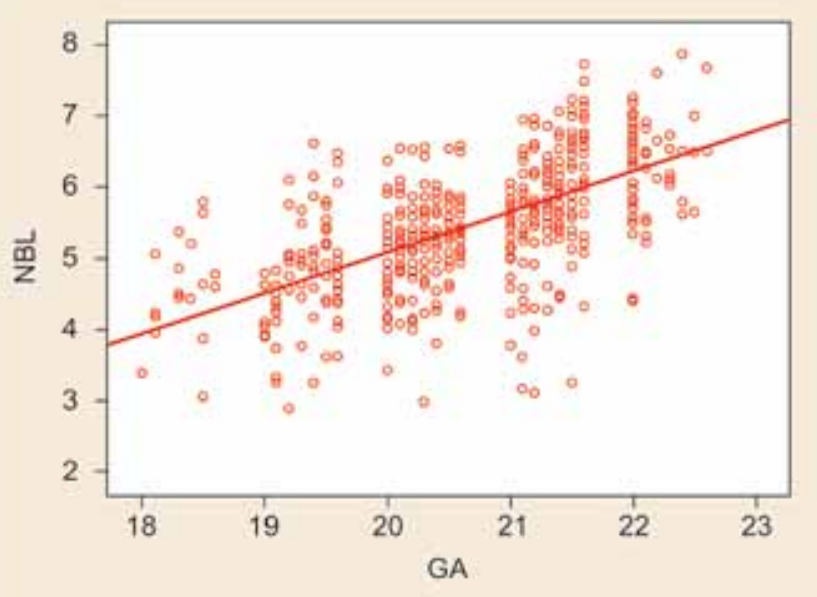

Graph 1: Scatter plot-gestational age vs mean nasal bone length
The 5th Centile NBL values correlated with the values from the study done by Sharma et al. ${ }^{10}$ The NBL value at 19 to 19.6 weeks are slightly less (3.5185 vs $3.8 \mathrm{~mm}$ ) and those at 21 to 21.6 and 22 to 22.6 weeks are more compared to those of Sharma et al (4.297 vs 4.2, 5.35 vs $4.4 \mathrm{~mm}$ ).

The 5th Centile NBL values differed from those of values obtained by Sonek et al. ${ }^{11}$ The 5 th Centile NBL lengths in Indian population are shorter compared to those of Caucasian population at all GA (18 to 22 weeks).

The 50th Centile of the NBL values with gestational age was compared with those from studies done by Sharma et $\mathrm{al}^{10}$ and Sonek et al. ${ }^{11}$ The 50th Centile NBL values correlated with those values of the Study done by Sharma et al. The NBL value at 19 to 19.6 weeks are slightly less (4.755 vs $4.9 \mathrm{~mm}$ ) and those at 20 to 22.6 weeks are more compared to those of Sharma et al (5.25 vs 5.2, 5.78 vs 5.6, 6.525 vs $6 \mathrm{~mm})$. The 50th Centile NBL values differed from those of values obtained by Sonek et al. The 50th Centile NBL lengths in Indian population are shorter compared to those of Caucasian population.

Similarly, the 95th Centile NBL values correlated with those values of the study done by Sharma et al. The NBL value at 19 to 19.6 to 21 to 21.6 weeks was slightly less (6.118 vs 6.3,6.09 vs 6.5 and 7.018 vs $7.2 \mathrm{~mm}$ ) compared to those of Sharma et al.

The 95th centile NBL values differed from those of Values obtained by Sonek et al. The NBL lengths in Indian population are shorter compared to those of Caucasian population.

The mean NBL values correlated with those values of the Study done by Sharma et al. The NBL value at 19 to 19.6 weeks was slightly less (4.756 vs $4.97 \mathrm{~mm}$ ) compared to those of Sharma et al and more at 21 to 21.6 and 22 to 22.6 weeks (5.78 vs 5.62 and 6.389 vs 5.96).

Table 1: Statistical measurements of the nasal bone lengths (All measurements in $\mathrm{mm}$ )

\begin{tabular}{llllllllll}
\hline GA-weeks & $N$ & 2.5th centile & 5th centile & 50th centile & 95th centile & Range & Median & SD & Mean \\
\hline $18-18.6$ & 19 & 3.2085 & 3.357 & 4.52 & 5.664 & $3.06-5.79$ & 4.52 & 0.709551 & 4.54 \\
$19-19.6$ & 75 & 3.2585 & 3.5185 & 4.755 & 6.118 & $2.9-4.62$ & 4.75 & 0.776394 & 4.756 \\
$20-21.6$ & 161 & 4.02 & 4.16 & 5.25 & 6.09 & $2.99-6.6$ & 5.25 & 0.646746 & 5.181 \\
$21-21.6$ & 169 & 3.815 & 4.297 & 5.78 & 7.018 & $3.11-7.05$ & 5.78 & 0.825733 & 5.788 \\
$22-22.6$ & 76 & 5.1225 & 5.35 & 6.525 & 7.625 & $4.4-7.87$ & 6.525 & 0.69789 & 6.389 \\
\hline
\end{tabular}

GA: Gestational age; SD: Standard deviation

Table 2: Results of comparable study from Indian population,Sharma et al ${ }^{10}$ and Caucasian population, Sonek et al ${ }^{11}$

\begin{tabular}{|c|c|c|c|c|c|c|c|c|}
\hline \multirow[t]{2}{*}{ Gestational age } & \multicolumn{2}{|c|}{$N$} & \multicolumn{2}{|c|}{ 5th centile } & \multicolumn{2}{|c|}{ 50th centile } & \multicolumn{2}{|c|}{ 95th centile } \\
\hline & Sonek et al & Sharma et al & Sonek et al & Sharma et al & Sonek et al & Sharma et al & Sonek et al & Sharma et al \\
\hline $18-18+6$ & 252 & 668 & 4.3 & 3.4 & 5.7 & 4.6 & 7 & 5.8 \\
\hline $19-19+6$ & 388 & 1237 & 5 & 3.8 & 6.3 & 4.9 & 7.9 & 6.3 \\
\hline $20-20+6$ & 440 & 1009 & 5.2 & 4.05 & 6.7 & 5.2 & 8.3 & 6.5 \\
\hline $21-21+6$ & 322 & 870 & 5.6 & 4.2 & 7.1 & 5.6 & 9 & 7.2 \\
\hline $22-22+6$ & 208 & 928 & 5.8 & 4.4 & 7.5 & 67. & 9.3 & 6 \\
\hline $23-23+6$ & & 72 & 6.4 & 4.8 & 7.9 & 6.3 & 9.6 & 7.83 \\
\hline
\end{tabular}




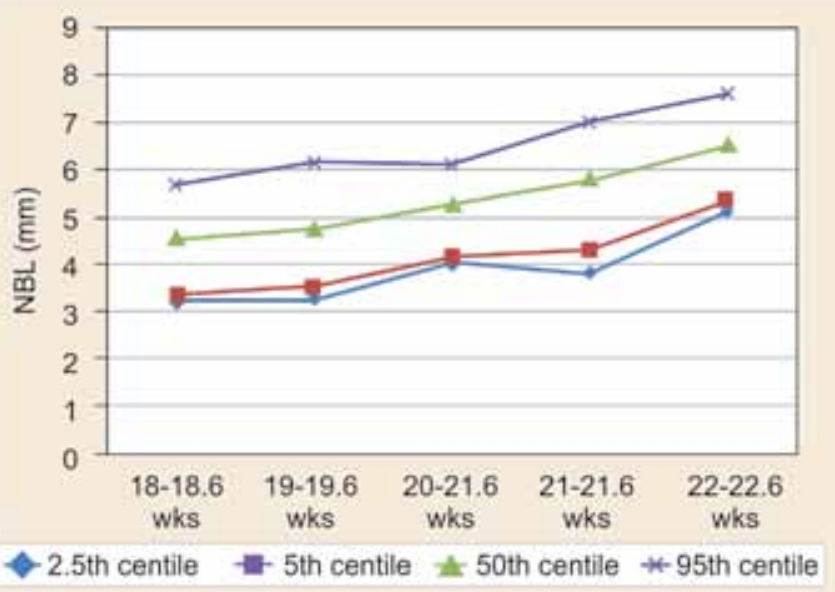

Graph 2: Line diagram comparing gestational age and nasal bone length values

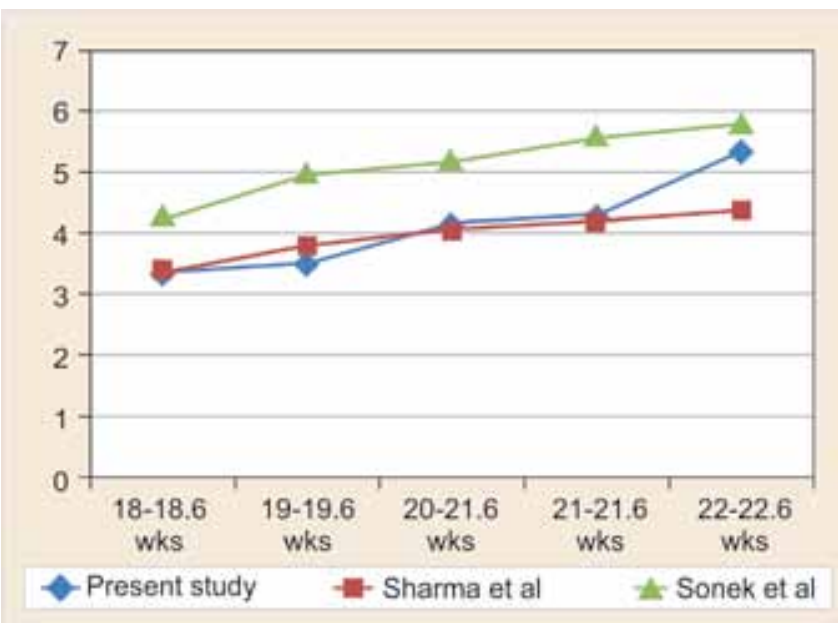

Graph 3: Line diagram comparing gestational age with 5th percentile nasal bone length (NBL) values of Sharma et $\mathrm{al}^{10}$ and Sonek et $\mathrm{al}^{11}$ : line diagram of the 5 th centile of the NBL values with gestational age comparing those with studies done by Sharma et al and Sonek et al. The 5th centile NBL values correlated with those values of the study done by Sharma et al. The NBL value at 19 to 19.6 weeks are slightly less ( $3.5185 \mathrm{vs} 3.8 \mathrm{~mm}$ ) and those at 21 to 21.6 and 22 to 22.6 weeks are more compared to those of Sharma et al (4.297 vs 4.2, 5.35 vs $4.4 \mathrm{~mm}$ )

The mean NBL values differed from those of values obtained by Gamez et al. ${ }^{12}$ The NBL lengths in Indian population are shorter compared to those of Caucasian population.

Correlation analysis was performed and yielded the following results:

1. The NBL and GA are positively correlated $(r=+0.626)$.

2. There is moderate correlation between NBL and GA.

3. The relationship is significant ( $p$-value $<0.001$ ).

4. The covariance of 0.581 suggests that the variables GA and NBL are positively correlated.

A regression analyis was performed and yielded the following equation:

Regression equation: $\mathrm{NB}=(0.5708 \times \mathrm{GA})-6.329$.

\section{DISCUSSION}

Though nasal bone is more frequently present in DS fetuses during second trimester than in the first trimester, a greater proportion of them are hypoplastic. ${ }^{13}$ Strong likelihood ratios and sound positive predictive values warrant NBL documentation during second trimester. Though interpretation was done traditionally based on centiles, recent studies showed improved efficacy with Multiple of Medians (MoMs). ${ }^{14}$

In countries, such as India where often only the second trimester scan is performed for screening of aneuploidies, NBL could be regarded as a useful marker. Prenatal screening for fetal aneuploidies is not yet incorporated in our national health program, which means that the majority of the population is screened for fetal chromosomal abnormalities mainly at the time of the anomaly scan. This therefore remains a form of primary prenatal screening in many of the Indian centers. ${ }^{10}$

By studying the values of NBLs in the fetuses of a South Indian population, a normal reference curve was drawn in our study. The values closely correlated with a similar study conducted by Sharma et $\mathrm{l}^{10}$ which comprised of both the south and north Indian population.

Nasal bone length has considerable variation with ethnicity as is seen with any anthropometric measurement. The fetal NBL in Chinese population appeared shorter than that of Caucasian and African-Americans. ${ }^{11,15}$ NBL in Thai fetuses at 11 to $13+6$ weeks GA was found to be on average shorter than that in Caucasian, AfricanAmerican and Chinese populations, but similar to those reported in Korean and Latin-American populations. ${ }^{16}$ The NBL was found to be shorter in Korean fetuses than Caucasian and Chinese fetuses. ${ }^{17}$ Nasal bone length in Japanese population was significantly shorter and BPD/ Nasal bone length was significantly greater than those in the Caucasian and black populations. ${ }^{18}$

In our study the NBLs of the fetuses at 18 to 22 weeks were significantly shorter to those of the Caucasian population. This is an important observation as the interpretation of NBL for an Indian fetus should not be from normograms based on western population which are widely available. The 5th centile for different GAs in Indian fetuses is significantly less than in Caucasian population. A lot of false positive cases can be avoided thereby reducing invasive tests hence averting a significant number of procedure related adverse risks.

Though all the measurements were performed by an experienced fetomaternal medicine consultant; the validation of this reference curve would further have to be carried out by studying a larger population and finding out the sensitivity for detection of DS using these 
reference values. Large population based data can be converted to MoMs values aiding improved interpretations. Statistical measures of performance (sensitivity and false positive values) of hypoplastic nasal bone based on such normograms needs to be investigated.

Also, it needs to be assessed whether there are any changes in the lengths in the different populations within India. Nasal bone length can form an effective screening tool in combination with the serum markers for DS by incorporating the adjustment according to the ethnicity.

\section{REFERENCES}

1. Sherman SL, Allen EG, Bean LH, Freeman SB. Epidemiology of Down syndrome. Ment Retard Dev Disabil Res Rev 2007; 13:221-227.

2. Bromley B, Lieberman E, Shipp T, Benacerraf B. Fetal nasal bone length: a marker for down syndrome in the second trimester. J Ultra Med 2002;21:1387-1394.

3. Nicolaides KH, Azar G, Byrne D. Fetal nuchal translucency: Ultrasound screening for chromosomal defects in first trimester of pregnancy. Br Med J 1992;304:867-889.

4. Sonek J, Cicero S, Neiger R. Nasal bone assessment in prenatal screening for trisomy 21. Am J Obstet Gynecol 2006;195: 1219-1230.

5. Sonek JD, Glover M, Zhou M, Nicolaides KH. Donald School J Ultra Obstet Gynecol 2010;4(2):97-116.

6. Shipp TD, Benacerraf BR. Second trimester ultrasound screening for chromosomal abnormalities. Prenat Diagn 2002;22(4):296-230.

7. Guis F, Ville Y, Doumerc S, Pons J, Frydman R. Ultrasound evaluation of the length of the fetal nasal bones throughout gestation. Ultra Obstet Gynecol 1995;5:304-307.

8. Cicero S, Sonek JD, Mckenna DS. Nasal bone hypoplasia in trisomy 21 at 15-22 weeks' gestation. Ultra Obstet Gynecol 2003;21:15-18.
9. Spencer K, Ong CY, Liao AW, Nicolaides KH. The influence of ethnic origin on first trimester biochemical markers of chromosomal. Prenat Diagn 2000;20(6):491-494.

10. Sharma A, Tayal T, Bh N, Radhakrishnan P, Kaul A. Evaluation of the reference values for the fetal nasal bone length at 16 to 25 weeks of gestational age in an Indian population. Prenat Diagn 2013;33(8):800-803.

11. Sonek J, McKenna D, Webb D, Croom C, Nicolaides KH. Nasal bone length throughout gestation: normal ranges based on 3537 fetal ultrasound measurements. Ultra Obstet Gynecol 2003;21:152-115.

12. Gamez F, Ferreiro P, Salmean JM. Ultrasonographic measurement of fetal nasal bone in a low-risk population at 19 to 22 gestational weeks. Ultra Obstet Gynecol 2003;23:152-153.

13. Cusick W, Shevell T, Duchan LS, Lupinacci CA, Terranova J, Crombleholme WR. Likelihood ratios for fetal trisomy 21 based on nasal bone length in the second trimester: how best to define hypoplasia? Ultra Obstet Gynecol 2007;30: 271-274.

14. Odibo AO, Sehdev HM, Stamilio DM, Cahill A, Dunn L, Macones GA. Defining nasal bone hypoplasia in secondtrimester Down syndrome screening: does the use of multiples of the median improve screening efficacy? Am J Obstet Gynecol 2007;197(4):361.e1-361.e4.

15. Chen M, Lee CP, Leung KY, Hui PW, Tang MHY. Pilot study on the midsecond trimester examination of fetal nasal bone in the Chinese population. Prenat Diagn 2004;24(2):87-91.

16. SutthibenjakulS,Suntharasaj T,SuwanrathC,Kor-AnantakulO, Geater A. A Thai reference for normal fetal nasal bone length at 15 to 23 weeks' gestation. J Ultra Med 2009; 28(1):49-53.

17. Jung E, Hye-Sung Won, Lee PR, Kim A. Ultrasonographic measurement of fetal nasal bone length in the second trimester in Korean population. Prenat Diagn 2007;27(2): 154-157.

18. Kanagawa T, Fukuda H, Kinugasa $Y$, Son M, Shimoya $K$, Murata Y, Yokoyama T, Hashimoto H, Isaka S, Nagamatsu M. Mid-second trimester measurement of fetal nasal bone length in the Japanese population. J Obstet Gynaecol Res 2006;32(4):403-407. 